Check for updates

Cite this: J. Mater. Chem. C, 2020 8, 12148

Received 19th May 2020,

Accepted 24th July 2020

DOI: $10.1039 / d 0 t c 02399 j$

rsc.li/materials-c

\title{
A fast response, self-powered and room temperature near infrared-terahertz photodetector based on a $\mathrm{MAPbl}_{3} / \mathrm{PEDOT}$ :PSS composite $\uparrow$
}

\author{
Yifan Li, (D) Yating Zhang, (D)* Tengteng Li, Xin Tang, Mengyao Li, Zhiliang Chen, \\ Qingyan Li, (D) Quan Sheng, Wei Shi and Jianquan Yao*
}

\begin{abstract}
Organic-inorganic halide perovskites with low thermal conductivity, high Seebeck coefficients and high carrier mobilities are promising thermoelectric materials for near infrared (NIR) and terahertz (THz) photodetectors (PDs). Here, we report a novel rapid response and self-powered $\mathrm{NIR}$ and $\mathrm{THz}$ photothermoelectric PD based on a $\mathrm{CH}_{3} \mathrm{NH}_{3} \mathrm{Pbl}_{3}\left(\mathrm{MAPbl}_{3}\right)$ and poly(3,4-ethylenedioxythiophene):poly(4styrenesulfonate) (PEDOT:PSS) composite. An order of magnitude enhancement in the Seebeck coefficient was observed that resulted from the addition of PEDOT:PSS. Under $1064 \mathrm{~nm}$ and $2.52 \mathrm{THz}$ illumination, the device displays a stable and repeatable photoresponse at room temperature under a zero bias voltage. The frequency response shows a $-3 \mathrm{~dB}$ frequency band of $5 \mathrm{kHz}$, corresponding to a fast response time of $28 \mu \mathrm{s}$, which is approximately three orders of magnitude faster than the previously reported results. These results demonstrate that $\mathrm{MAPb}_{3} / \mathrm{PEDOT}$ :PSS is a promising composite material for fast response and self-powered NIR-THz PTE PDs operating at room temperature.
\end{abstract}

\section{Introduction}

Broadband photodetectors (PDs) that offer operation over the range from the near infrared (NIR) to terahertz (THz) region in a single device with simple structures are promising for applications including imaging, remote sensing, communications and spectroscopy. ${ }^{1-3}$ In particular, with the continuing development of $\mathrm{THz}$ technology, self-powered, fast response and room temperature $\mathrm{THz}$ PDs are urgently required for space network communication and biomedical imaging. ${ }^{4-6}$ However, the current commercial detectors can hardly meet these requirements; for example, the detection range of Ge photodiodes is limited to 400-2000 nm, the Bolometer must work at low temperature (1.5-4.2 K), Golay cells always show a slow response time of about $100 \mathrm{~ms}$ and Schottky diodes can only detect frequency below $0.33 \mathrm{GHz}$. To date, many physical mechanisms have been found to be responsible for the photoresponse in broadband PDs, including the photoconductive (PC) effect, photovoltaic (PV) effect, and thermoelectric effects (bolometric, photothermoelectric (PTE)

Key Laboratory of Optoelectronics Information Technology, Institute of Laser \& Opto-Electronics, School of Precision Instruments and Optoelectronics Engineering, Tianjin University, Tianjin 300072, China. E-mail: yating@tju.edu.cn $\dagger$ Electronic supplementary information (ESI) available: The AFM surface morphology of the $\mathrm{MAPbI}_{3}$ film, the PL spectra of the $\mathrm{MAPbI}_{3} / \mathrm{PEDOT}$ :PSS thin film, the $I-V$ curves in a wider range of $-1 \mathrm{~V}$ to $1 \mathrm{~V}$ bias voltage, the $D$ curve under $1064 \mathrm{~nm}$ and $2.52 \mathrm{THz}$ irradiation as a function of power intensity, and the Seebeck coefficient of the $\mathrm{MAPbI}_{3} / \mathrm{PEDOT}$ :PSS device. See DOI: 10.1039/d0tc02399j and pyroelectric effects). ${ }^{7-10}$ It is well known that PC and PV effects are limited to a detection range in which the photon energy is larger than the band gap of the effective material. In contrast, thermoelectric effects are related to photoinduced hot carriers, which are independent of the incident light wavelength. ${ }^{11}$

PDs based on the PTE effect also called the Seebeck effect have shown potential applications in broadband detection benefiting from their simple synthesis strategies, self-powered ability, low power consumption and room temperature operation, which makes these devices good candidates for NIR-THz detection. ${ }^{12,13}$ In recent years, many semiconductor nanomaterials have been considered as potential light-sensitive materials for PTE PDs. ${ }^{10,13-15}$ Typically, graphene-based PTE PDs have been studied widely, including single-bilayer interface junction formation, antenna array structure construction, and the preparation of reduced graphene oxide..$^{12,16,17}$ In our recent work, we have reported the PTE photoresponses of three-dimensional graphene foam (3D GF) ${ }^{3}$ and reduced graphene oxide/ $\mathrm{CsPbrBr}_{3}{ }^{18}$ However, limited by their complex structure and slow response time, graphene-based PTE PDs are still hardly used. Additionally, in view of the fact that previously reported PTE devices with millimetric channels have presented slow response times $\left(>1 \mathrm{~ms}\right.$ ), fast response PTE PDs are urgently required. ${ }^{3,12,13}$ Therefore, it is desirable to explore novel thermal materials for PTE PDs.

In recent years, organic-inorganic hybrid perovskite $\mathrm{CH}_{3} \mathrm{NH}_{3} \mathrm{PbI}_{3}$ $\left(\mathrm{MAPbI}_{3}\right)$ with the characteristics of a wide absorption range, large light absorption coefficient and high carrier mobility has been demonstrated to exhibit considerable superiority in ultraviolet 
(UV)-visible (Vis) range optoelectronic devices and solar cells. ${ }^{19-23}$ In our recent work, ${ }^{24} \mathrm{MAPbI}_{3}$ was demonstrated to offer a high Seebeck coefficient and low thermal conductivity, which are both desirable properties for thermoelectric materials. ${ }^{25,26}$ Moreover, Ling Xu's group investigated the Seebeck effect in $\mathrm{MAPbI}_{3}$ polycrystalline thin films and they demonstrated that the low conductivity of these films can be beneficial for heat harvesting and proved a large temperature gradient. ${ }^{26}$ In general, the most effective strategy is to construct a heterojunction using two materials with different Seebeck coefficients to improve the overall PTE response. ${ }^{16,27}$ Polymer-based PTE PDs such as poly(3,4-ethylenedioxythiophene):poly(styrenesulfonate) (PEDOT: PSS) devices are receiving increasing attention because of their excellent thermoelectric properties, solution processing capability and high flexibility. ${ }^{27-30}$ The high Seebeck coefficient of up to $436 \mu \mathrm{V} \mathrm{K}^{-130}$ and high electrical conductivity of up to $\sim 10^{4} \mathrm{~S} \mathrm{~m}^{-1}$ of PEDOT:PSS ${ }^{28}$ can be beneficial for electron transmission in PTE devices.

In this work, we fabricated a self-powered, high response speed and room temperature PTE PD using a $\mathrm{MAPbI}_{3} / \mathrm{PEDOT}$ :PSS composite that can be operated in both NIR and $\mathrm{THz}$ ranges. The photoelectric testing demonstrates that this $\mathrm{MAPbI}_{3} / \mathrm{PEDOT}$ : PSS PD exhibits stable and repeatable photoresponses under both $1064 \mathrm{~nm}$ and $2.52 \mathrm{THz}$ irradiation at room temperature. As a result, the device shows a fast response time of $28 \mu$ s under a zero bias voltage. In addition, the relationship between the temperature distribution and photocurrent confirms that the PTE effect dominates the photocurrent generation in the device. Although the performance of the $\mathrm{MAPbI}_{3} / \mathrm{PEDOT}$ :PSS PD needs to be improved, it still provides a new approach to the construction of NIR-THz broadband detectors.

\section{Results and discussion}

Fig. 1a shows a schematic of the $\mathrm{MAPbI}_{3} / \mathrm{PEDOT}$ PSS PD. Details of the device fabrication process are provided in the
Methods section. The cross-sectional morphology of the device was measured by scanning electron microscopy (SEM), with the results shown in Fig. 1b. The multilayers composed of $\mathrm{Au}$ $(\sim 60 \mathrm{~nm}), \mathrm{MAPbI}_{3}(\sim 130 \mathrm{~nm})$, PEDOT:PSS $(\sim 40 \mathrm{~nm})$ and ITO $(\sim 1800 \mathrm{~nm})$ can be seen clearly in the cross-sectional image. The surface morphology of the $\mathrm{MAPbI}_{3} / \mathrm{PEDOT}$ :PSS film was obtained by using SEM, as shown in Fig. 1c. The morphology shows numerous uniform grain boundaries that are closely packed with neighboring grains. An average gran size of about 60-70 $\mathrm{nm}$ was observed in the atomic force microscopy (AFM) surface morphology of the $\mathrm{MAPbI}_{3}$ film, as shown in Fig. S1. (ESI $\dagger$ ). The crystal structure of the $\mathrm{MAPbI}_{3} / \mathrm{PEDOT}$ :PSS thin film was characterized via X-ray diffraction (XRD) measurement, as shown in Fig. 1d. The XRD pattern exhibits the main diffraction peaks at $2 \theta=14.1^{\circ}, 28.42^{\circ}, 31.86^{\circ}$ and $43.18^{\circ}$, which match with the (110), (220), (310), and (330) crystallographic planes of $\mathrm{MAPbI}_{3},{ }^{31,32}$ respectively. Fig. 1e shows the broadband absorption spectra of the $\mathrm{MAPbI}_{3}$, PEDOT:PSS and $\mathrm{MAPbI}_{3} / \mathrm{PEDOT}$ :PSS thin films over the range from 400 to $2200 \mathrm{~nm}$. The $\mathrm{MAPbI}_{3}$ and $\mathrm{MAPbI}_{3} / \mathrm{PEDOT}$ :PSS films exhibit strong absorption characteristics within the UV and visible range from 400 to $780 \mathrm{~nm}$ and weak absorption characteristics in the NIR range from 780 to $2200 \mathrm{~nm}$. This can be explained as follows. In the wavelength range from 400 to $780 \mathrm{~nm}$, because the incident light energy is larger than that of the $\mathrm{MAPbI}_{3}$ and $\mathrm{MAPbI}_{3} / \mathrm{PEDOT}$ :PSS films' band gap, the absorption of photon energy is mainly dependent on the electron interband transition and thus the PD shows high absorption. In contrast, at wavelengths beyond $780 \mathrm{~nm}$, the laser energy is lower than that of the optical band gap. In this case, the PD mainly relies on hot electrons to absorb photon energy, which results in low absorption. The PEDOT:PSS absorption spectrum shows an increasing absorbance from UV to NIR, which is consistent with previous results. ${ }^{29}$ The photoluminescence (PL) spectrum of the device is shown in Fig. S2 (ESI $\dagger$ ). Fig. 1f shows the TDS absorption spectra of the $\mathrm{MAPbI}_{3}$, PEDOT:PSS and $\mathrm{MAPbI}_{3} / \mathrm{PEDOT}$ :PSS thin films in the $\mathrm{THz}$ range. Broadband $\mathrm{THz}$ absorption is obtained
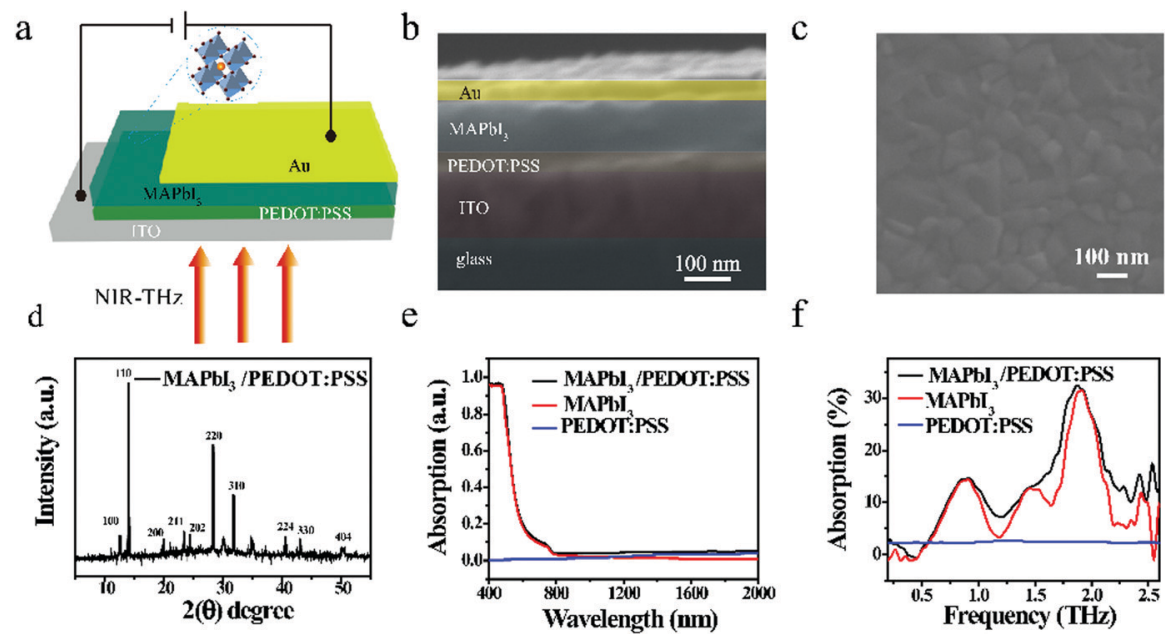

Fig. 1 (a) Schematic illustration of the MAPb| 3 /PEDOT:PSS PD. (b) Cross-sectional image of the device with the structure of ITO/PEDOT:PSS/MAPbl $3 /$ Au. (c) Surface SEM image of the MAPbI 3 /PEDOT:PSS film. (d) XRD pattern of the MAPbI 3 /PEDOT:PSS film. (e) UV-NIR absorption spectra of MAPbI

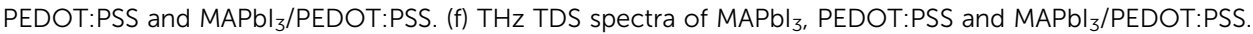


within 0.2 to $2.59 \mathrm{THz}$. Two strong absorption peaks at 0.89 and $1.87 \mathrm{THz}$ with absorbance of $14 \%$ and $32 \%$, respectively, can be clearly observed in the $\mathrm{MAPbI}_{3}$ and $\mathrm{MAPbI}_{3} / \mathrm{PEDOT}$ :PSS thin films. PEDOT:PSS shows a relatively low and stable absorption spectrum within the range from 0.2 to $2.59 \mathrm{THz}$. The absorption results reveal that $\mathrm{MAPbI}_{3}$ is a good light harvester over the UV to $\mathrm{THz}$ range.

The photoelectric characteristics of the $\mathrm{MAPbI}_{3} / \mathrm{PEDOT}$ :PSS PD were investigated under dark conditions and at different laser powers at $1064 \mathrm{~nm}$ and $2.52 \mathrm{THz}$. The $I-V$ curves obtained from the $\mathrm{MAPbI}_{3} / \mathrm{PEDOT}$ :PSS PD under $1064 \mathrm{~nm}$ and $2.52 \mathrm{THz}$ laser irradiation at different laser power densities are shown in Fig. 2a and b, respectively. It is obvious that in the small bias voltage range, the $I-V$ curves exhibit an Ohmic characteristic and the curves shift upon light illumination, which are typical PTE effect features. ${ }^{13,33}$ The curves indicate that photocurrent increases with increasing laser power intensity in the $-5 \mathrm{mV}$ to $5 \mathrm{mV}$ range of bias voltage. The $I-V$ curves obtained over a wider bias voltage range from $-1 \mathrm{~V}$ to $1 \mathrm{~V}$ are shown in Fig. S3 (ESI $\dagger$ ). Under excitation of a large bias voltage, a Schottky barrier was formed in the device, because of different functions, which is consistent with the previously reported results. ${ }^{26}$ To quantify the junction current of the device under light illumination, logarithmic $I-V$ characteristics of the device under $1064 \mathrm{~nm}$ and 2.52 THz illumination are shown in Fig. 2c and d, respectively. As shown in Fig. $2 \mathrm{c}$ and $\mathrm{d}$, under dark conditions, $\mathrm{a}-0.31 \mathrm{~V}$ open circuit voltage $V_{\text {oc }}$ can be observed. And a net photocurrent is generated by the built in field even at zero bias.
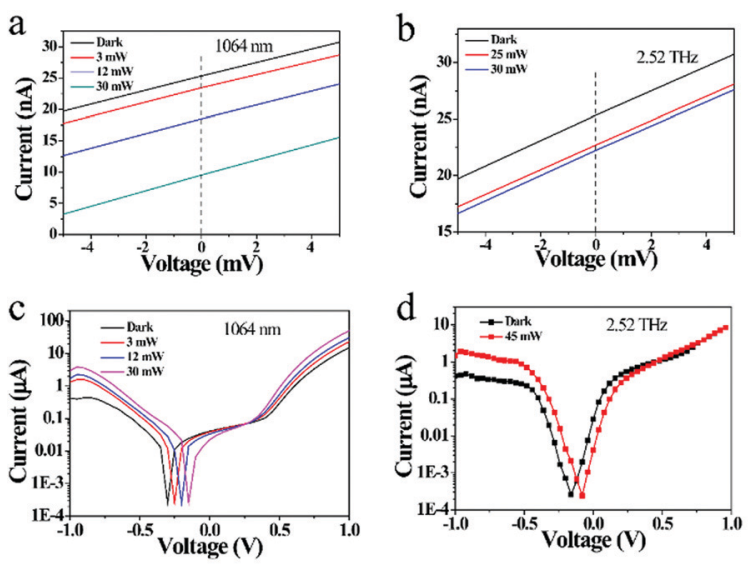

e

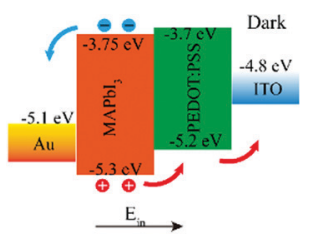

f

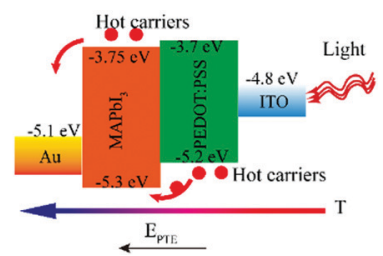

Fig. 2 Current-voltage $(I-V)$ characteristics of the MAPb| ${ }_{3} /$ PEDOT:PSS device in the dark and under light illumination with different power intensities at (a) $1064 \mathrm{~nm}$ and (b) $2.52 \mathrm{THz}$. The logarithmic I-V characteristics of the device in the dark and under light illumination with different power intensities at (c) $1064 \mathrm{~nm}$ and (d) $2.52 \mathrm{THz}$. Schematic of the energy band and charge transfer at the $\mathrm{MAPb}_{3} / \mathrm{PEDOT}$ :PSS interface (e) in the dark and (f) under light illumination.
With the laser power increasing, the $V_{\text {oc }}$ shifts to a low negative voltage, which could be attributed to the generation of PTE voltage. Moreover, with increasing power intensity, the shortcircuit current $I_{\mathrm{sc}}$ changed, indicating an increase in the number of hot carriers under light illumination. The photocurrent generation mechanism is discussed fully here. Fig. 2e illustrates the band energy of the device and the charge transfer under dark conditions. Holes transfer towards the PEDOT:PSS layer while electrons tend to drift to the Au electrode, because of the different functions of the different layers. A built in field $E_{\text {in }}$ forms in the device and its direction is from the Au electrode to the ITO electrode. This $E_{\text {in }}$ direction of the device is opposite to the external circuit, as shown in Fig. 1a, and this results in the negative $V_{\text {oc }}$ being obtained under dark conditions. Under light illumination on the ITO electrode, a photo induced temperature gradient is produced, as shown in Fig. 2f. Upon thermal excitation, the hot carriers are transported from the PEDOT:PSS layer to the $\mathrm{MAPbI}_{3}$ layer, forming a Seebeck field, which is also called a PTE field $E_{\mathrm{PTE}}$. The direction of $E_{\mathrm{PTE}}$ points from the ITO electrode towards the Au electrode. This PTE field is opposite in orientation to the built-in field, which leads to the positive shift of $V_{\mathrm{oc}}$. In this device, the photocurrent generation is mainly determined by the hot carrier transport. According to the Seebeck effect, the PTE voltage can be defined as $\Delta V=\Delta S \times \Delta T$, where $\Delta S$ is the Seebeck difference between the different layers and $\Delta T$ is the temperature gradient of the device under light irradiation.

Additionally, the photoresponses of the $\mathrm{MAPbI}_{3} / \mathrm{PEDOT}$ :PSS PD were investigated under irradiation at $1064 \mathrm{~nm}$ and $2.52 \mathrm{THz}$. Fig. 3a shows the time-dependent photocurrent responses of the device produced by periodically turning the light illumination on and off under $1064 \mathrm{~nm}$ laser illumination at $0 \mathrm{~V}$ bias voltage. It is obvious that the device photocurrent increased with increasing power, thus demonstrating good on/off switching behaviors.
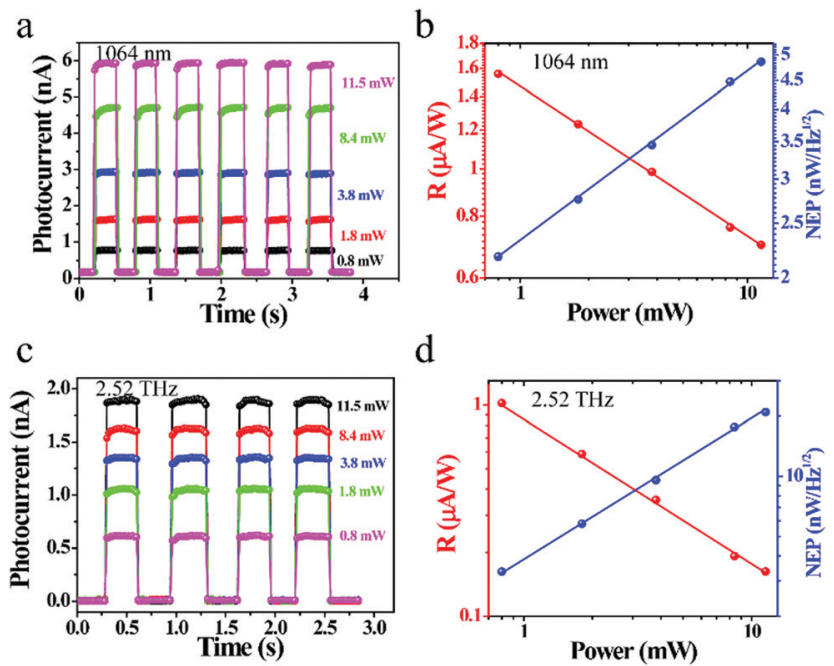

Fig. 3 (a) Time-dependent photocurrents of the device under switching on/off $1064 \mathrm{~nm}$ illumination. (b) $R$ and NEP of the device as a function of $1064 \mathrm{~nm}$ laser power intensity. (c) Time-dependent photocurrents of the devices under switching on/off $2.52 \mathrm{THz}$ illumination. (d) $R$ and NEP of the device as a function of $2.52 \mathrm{THz}$ laser power intensity. 
The photocurrent increases from $0.8 \mathrm{nA}$ to $6 \mathrm{nA}$ when the incident light intensity varies from $0.8 \mathrm{~mW}$ to $11.5 \mathrm{~mW}$. Moreover, the time-dependent photocurrents exhibit stable and repeatable characteristics, thus indicating the excellent stability of the device under $1064 \mathrm{~nm}$ laser irradiation. According to the obtained photocurrents under different laser power, the device responsivity $(R)$ was calculated by the formula (1):

$$
R=\frac{I_{\mathrm{ph}}}{P}=\frac{I_{\mathrm{ph}}}{E_{\mathrm{e}} \times A}
$$

Here, $I_{\mathrm{ph}}$ is the photocurrent, and $P, E_{\mathrm{e}}$ and $A$ are the incident laser power, irradiance density and the effective channel area, respectively. Accordingly, the detectivity $\left(D^{*}\right)$, and the noise equivalent power (NEP) can be calculated by the following equations, respectively:

$$
\begin{gathered}
D^{*}=R \sqrt{A} /\left(2 e I_{\text {dark }}\right)^{1 / 2} \\
\mathrm{NEP}=\sqrt{ } A / D^{*}
\end{gathered}
$$

where $e$ is the electron charge. The $R$ and NEP curves at $0 \mathrm{~V}$ bias voltage as a function of laser power are shown in Fig. 3b. The $R$ value decreases and the NEP value increases with increasing power. Therefore, the highest $R$ and lowest NEP can be measured at the lowest irradiation laser power. As shown in Fig. 3b, under $0.8 \mathrm{~mW}$ light illumination, the highest $R$ is $1.6 \mu \mathrm{A} \mathrm{W}^{-1}$ and the lowest NEP is $2.1 \mathrm{nW} \mathrm{Hz}{ }^{-1 / 2}$. In addition, the $R$ and NEP curves present a linear relationship with the power, which is consistent with the previous reported results. ${ }^{3}$

Fig. 3c shows the optical switched on/off photocurrents of the device under $2.52 \mathrm{THz}$ irradiation with varying power intensities at $0 \mathrm{~V}$ bias voltage. The photocurrents present stable and repeatable characteristics under different power intensities and increase from 0.6 to $1.8 \mathrm{nA}$ with increasing laser power from 0.8 to $11.5 \mathrm{~mW}$. Similarly, the photocurrents illustrate the good stability of the device under $2.52 \mathrm{THz}$ laser irradiation. $R$ and NEP are both plotted as a functions of power intensity in Fig. 3d. It shows that the $R$ curve displays a decreasing trend while the NEP curve shows an increasing tendency with increasing power intensity, revealing the highest $R$ value of $1.0 \mu \mathrm{A} \mathrm{W}^{-1}$ and the lowest NEP value of $3.3 \mathrm{nW} \mathrm{Hz}{ }^{-1 / 2}$. $R$ and $P$ present a linear relationship when plotted in the double-logarithmic coordinates and can be expressed as $\log R \sim \alpha \log P$, where $\alpha$ is a parameter without units. According to the reported results, the PTE photocurrent has a nonlinear dependence of $1 / T^{\beta-1},{ }^{10}$ while $T$ has a power law dependence on $P^{\gamma}{ }^{3}$ Then, $R$ can be expressed as $\log R \sim \alpha \log P$. The $D$ curves with units of $\mathrm{cm} \mathrm{Hz}^{1 / 2} \mathrm{~W}^{-1}$ under irradiation at $1064 \mathrm{~nm}$ and $2.52 \mathrm{THz}$ are plotted as a function of power intensity in Fig. S4 (ESI $\dagger$ ).

Based on the photoresponses obtained under NIR and THz illumination, the photocurrent generation mechanism can now be discussed fully. Many physical mechanisms may be responsible for the photoresponse of the $\mathrm{MAPbI}_{3} / \mathrm{PEDOT}$ :PSS PD, including the PC effect, PV effect, bolometric effect, pyroelectric effect and PTE effect. PC and PV effects are limited to the detection wavelength range wherein the photon energies are larger than the band gap of the effective material. ${ }^{11}$ The photon energy of NIR and THz is smaller than the $1.5 \mathrm{eV}$ energy band gap of the device, as shown in Fig. 2e. Therefore, PC and PV effects cannot be used to explain the photocurrent generation. In contrast, thermoelectric effects are related to photoinduced hot carriers, which are independent of the incident wavelength. It is well known that the bolometric effect must work under the application of a bias voltage. ${ }^{8}$ Since the $\mathrm{MAPbI}_{3} / \mathrm{PEDOT} \mathrm{PSS} \mathrm{PD}$ a

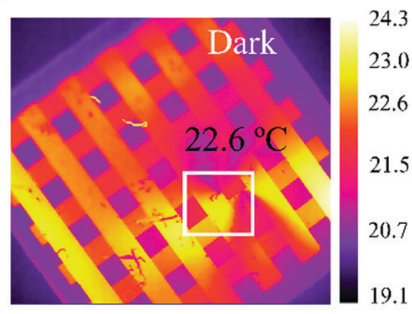

d

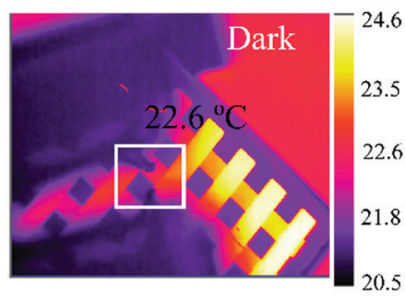

b

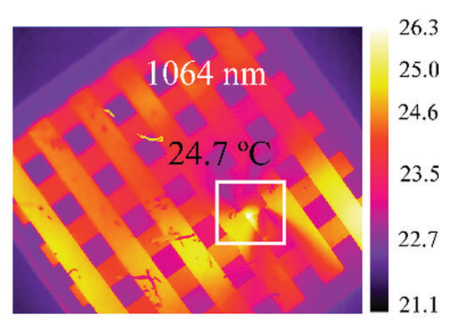

e

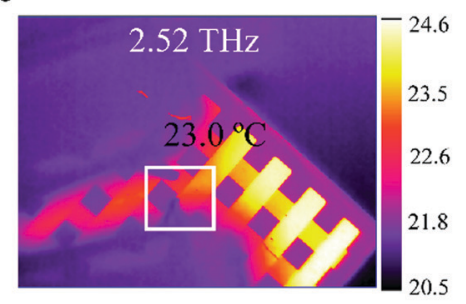

$\mathrm{C}$

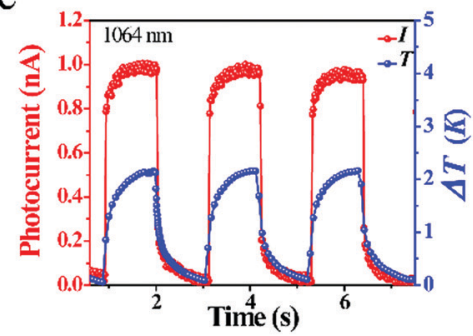

f

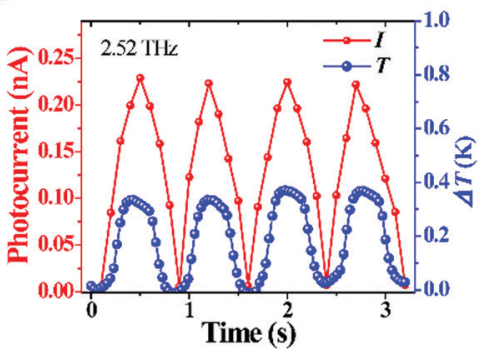

Fig. 4 Thermoelectric response mechanism of the device. (a and b) Temperature ( $T$ ) distributions of the device in the dark and under $1064 \mathrm{~nm}$ laser illumination $(0.8 \mathrm{~mW}$ ), respectively. (c) Photocurrent and $\Delta T$ of the device under switching on/off $1064 \mathrm{~nm}$ at $0.8 \mathrm{~mW}$. (d and e) Temperature ( $T$ ) distributions of the device in the dark and under $2.52 \mathrm{THz}$ laser illumination $(0.8 \mathrm{~mW})$, respectively. (f) Photocurrent and $\Delta T$ of the device under switching on/off $2.52 \mathrm{THz}$ at $0.8 \mathrm{~mW}$. 
operates at zero bias, the bolometric effect thus does not apply to the device. According to the time-dependent photocurrent characteristics of the device obtained when switching the $1064 \mathrm{~nm}$ and $2.52 \mathrm{THz}$ illumination on and off, which are shown in Fig. 3a and c, respectively, the pyroelectric effect can be excluded. ${ }^{34}$ Therefore, the PC, PV, bolometric and pyroelectric effects are eliminated, meaning that the PTE effect is responsible for the photoresponse of the device.

To aid in further understanding the PTE effect in our proposed device, temperature distribution and changes of the device under $1064 \mathrm{~nm}$ and $2.52 \mathrm{THz}$ light illumination were measured using a FLIR infrared imaging device. As shown in Fig. 4a and b, under dark conditions, the $\mathrm{MAPbI}_{3} / \mathrm{PEDOT}_{\mathrm{PSS}}$ PD shows a relatively low temperature of about $22.6{ }^{\circ} \mathrm{C}$. Under $1064 \mathrm{~nm}$ laser illumination at a power of $1 \mathrm{~mW}$, the surface temperature $\left(24.7{ }^{\circ} \mathrm{C}\right)$ increased by $2.1{ }^{\circ} \mathrm{C}$. The photocurrent and temperature gradient $\Delta T$ of the device measured under $1064 \mathrm{~nm}$ optical switching on/off at $0 \mathrm{~V}$ bias voltage are shown in Fig. 4c. The results show that over multiple optical switching cycles, the trend of photocurrent changes is consistent with that of temperature change. Similarly, the infrared images in Fig. $4 \mathrm{~d}$ and e clearly show the surface temperature distribution of the device under $2.52 \mathrm{THz}$ irradiation. The device shows a relatively low temperature of approximately $22.6{ }^{\circ} \mathrm{C}$ in the dark. Under $2.52 \mathrm{THz}$ laser illumination at a power of $0.3 \mathrm{~mW}$, the surface temperature $\left(23{ }^{\circ} \mathrm{C}\right)$ increased by $0.4{ }^{\circ} \mathrm{C}$. Furthermore, photocurrent at $0 \mathrm{~V}$ and temperature switching curves with the same tendency were acquired and are presented in Fig. 4f. As illustrated in Fig. 2f, upon light irradiation, a temperature gradient forms throughout the device; meanwhile, photocurrent generation depends on the hot carrier transport. The Seebeck coefficient values of $\mathrm{MAPbI}_{3}$, PEDOT:PSS and $\mathrm{MAPbI}_{3} / \mathrm{PEDOT}$ : PSS films are plotted as a function of temperature in Fig. S5 $(\mathrm{ESI} \dagger)$. By constructing a heterojunction structure, the Seebeck coefficient of the device increases greatly. The highest value of $\sim 532 \mu \mathrm{V} \mathrm{K}^{-1}$ at $45{ }^{\circ} \mathrm{C}$ is one order of magnitude higher than that of the pure $\mathrm{MAPbI}_{3}$ device. ${ }^{26}$ As shown in Fig. $4 \mathrm{c}$ and $\mathrm{f}$, the device displays $2 \mathrm{~K}$ and $0.4 \mathrm{~K}$ temperature differences under $1064 \mathrm{~nm}(1 \mathrm{~mW})$ and $2.52 \mathrm{THz}(0.3 \mathrm{~mW})$ illumination, respectively. According to the PTE effect, the photovoltage can be defined as $\Delta V=\Delta S \times \Delta T\left(\Delta V_{1064 \mathrm{~nm}}=1.05 \mathrm{mV}\right.$ and $\left.\Delta V_{2.52} \mathrm{THz}=0.199 \mathrm{mV}\right)$ under $1064 \mathrm{~nm}$ and $2.52 \mathrm{THz}$ illumination. The photocurrent can be expressed as $I=\Delta V / R_{\mathrm{h}}$, where $R_{\mathrm{h}}$ is the device resistance and the value of $R_{\mathrm{h}}$ is approximately $1 \times 10^{6} \Omega$, as shown in Fig. 2a and b. Then, the photocurrents of the device can be calculated to be $1 \mathrm{nA}$ and $0.2 \mathrm{nA}$ under $1064 \mathrm{~nm}(1 \mathrm{~mW})$ and $2.52 \mathrm{THz}(0.3 \mathrm{~mW})$ illumination, respectively, and these values are consistent with the experimental values given in Fig. $4 \mathrm{c}$ and $\mathrm{f}$. Therefore, both the experimental results and the theoretical analysis confirm that the PTE effect dominates the photocurrent generation in the $\mathrm{MAPbI}_{3} / \mathrm{PEDOT}$ :PSS PD.

Response speed is another important performance parameter for a PD. Fig. 5a shows a schematic diagram of the response time test system used for the device. The $1064 \mathrm{~nm}$ laser was modulated by a wave generator to produce specific frequency switching laser. A resistor with equivalent resistance to that of the $\mathrm{MAPbI}_{3} / \mathrm{PEDOT}$ :PSS PD is used in the series circuit. Then, the response time can be recorded by using an oscilloscope. Fig. 5b displays the normalized responsivity with respect to the modulation frequency under $1064 \mathrm{~nm}$ irradiation at $0 \mathrm{~V}$ bias voltage. The $-3 \mathrm{~dB}$ bandwidth of the device was acquired at $5 \mathrm{kHz}$. The relationship between the response time and the $-3 \mathrm{~dB}$ bandwidth frequency is shown below:

$$
t=\frac{1}{2 \pi f_{\mathrm{c}}}
$$

Here, $f_{\mathrm{c}}$ is the $-3 \mathrm{~dB}$ bandwidth frequency, and $t$ is the response time. ${ }^{35,36}$ Then, the response time can be calculated to be $31 \mu \mathrm{s}$. Multiple cycles of photocurrent response and response time under $1064 \mathrm{~nm}$ laser irradiation with the frequency of $5 \mathrm{kHz}$ are shown in Fig. $5 c$ and d, respectively. The device obviously shows stable and repeatable response characteristics at $5 \mathrm{kHz}$. As shown in Fig. 5d, the rise time can be defined as the time taken to increase from $10 \%$ to $90 \%$ of the maximum and the fall time can be defined as the time taken to decrease from $90 \%$ to $10 \%$ of the maximum. The $\mathrm{MAPbI}_{3} / \mathrm{PEDOT}$ :PSS PD shows fast rise and fall times of 28 and $33 \mu$ s, respectively. The results are three orders of magnitude faster than those of the previously reported PTE detectors. ${ }^{3,13}$

The Seebeck coefficients of $\mathrm{MAPbI}_{3}$, PEDOT:PSS and $\mathrm{MAPbI}_{3} /$ PEDOT:PSS under different light irradiation with $0.8 \mathrm{~mW}$ power are shown in Table S1 (ESI $\dagger$ ). The carrier concentration $(n)$, Hall mobility $\left(\mu_{\mathrm{H}}\right)$, and calculated conductivity $\left(\sigma_{\mathrm{H}}\right)$ of the $\mathrm{MAPbI}_{3} /$ PEDOT:PSS PD obtained by using a Hall test system are shown in Table S2 (ESI $\dagger$ ). Moreover, a comparison of the critical parameters of PEDOT:PSS based composite photodetectors is presented in Table $1 .^{27,37-40}$ And the comparison of the critical parameters of PTE photodetectors based on thermoelectric materials is presented in Table 2.,12,13,27,41 When compared with the reported results, the $\mathrm{MAPbI}_{3} / \mathrm{PEDOT}$ PSS PD displays the fastest response speed and a relatively high detectivity. Moreover, the detection band
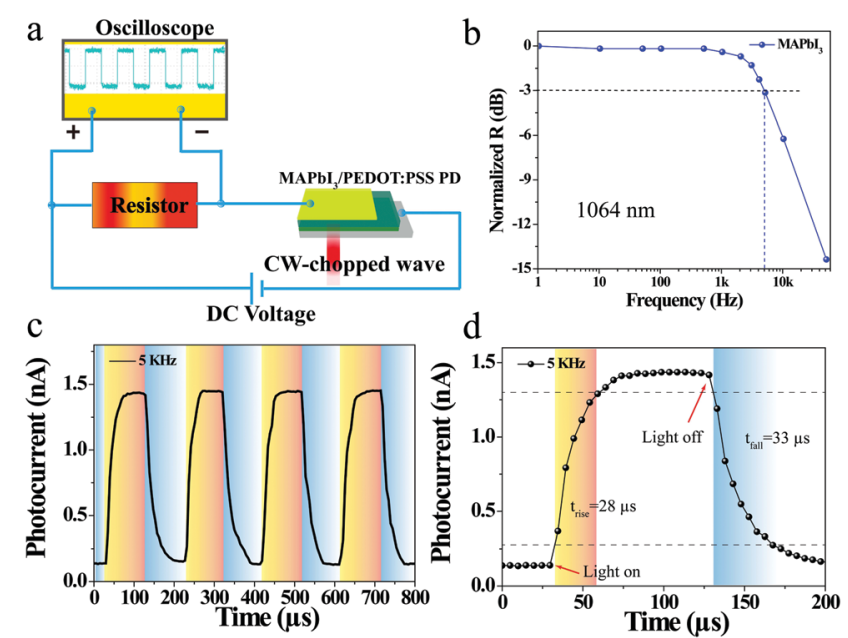

Fig. 5 (a) Schematic diagram of time response test system of the device. (b) Normalized responsivity as a function of modulation frequency under $1064 \mathrm{~nm}$ irradiation and the $f_{-3 \mathrm{~dB}}$ bandwidth of the device. Photocurrent response of the device under $1064 \mathrm{~nm}$ laser irradiation with the frequency of $5 \mathrm{kHz}$ : (c) multiple cycles and (d) one cycle with the rise and fall time. 
Table 1 Comparison of the critical parameters of PEDOT:PSS based composites photodetectors

\begin{tabular}{|c|c|c|c|c|c|}
\hline Description & Wavelength & Responsivity $\left(\mathrm{mA} \mathrm{W}^{-1}\right)$ & Detectivity (Jones) & Response time & Ref. \\
\hline PEDOT:PSS/graphene & $7.8 \mu \mathrm{m} \mathrm{IR}$ & $2.3 \mathrm{~V} \mathrm{~W}^{-1}(0 \mathrm{~V}$ bias $)$ & $1.4 \times 10^{7}$ & $20 \mathrm{~s}$ & 27 \\
\hline Al: MgZnO/PEDOT:PSS & $278 \mathrm{~nm}$ UV-B & $19.1 \mathrm{~mA} \mathrm{~W}^{-1}$ (0 V bias) & - & $3.5 \mathrm{~s}$ & 37 \\
\hline Single ZnO/PEDOT:PSS & 325 nm UV & $6.8 \times 10^{-3}(0 \mathrm{~V}$ bias $)$ & $9.3 \times 10^{9}$ & $4.5 \mathrm{~s}$ & 38 \\
\hline $\mathrm{MAPbI}_{3-x} \mathrm{Cl}_{x} /$ PEDOT:PSS & 598-895 nm UV-NIR & $1.91 \times 10^{9}(0.5 \mathrm{~V}$ bias $)$ & $1.4 \times 10^{14}$ & $>50 \mathrm{~s}$ & 39 \\
\hline PEDOT:PSS/ZnO & $300-400 \mathrm{~nm} \mathrm{UV}$ & $2.5 \times 10^{5}(-1 \mathrm{~V}$ bias $)$ & $2.5 \times 10^{11}$ & $0.94 \mathrm{~s}$ & 40 \\
\hline $\mathrm{MAPbI}_{3} / \mathrm{PEDOT}: \mathrm{PSS}$ & $1064 \mathrm{~nm}-118 \mu \mathrm{m}$ NIR-THz & $1.6 \times 10^{-3}(0 \mathrm{~V}$ bias $)$ & $1.2 \times 10^{7}$ & $28 \mu \mathrm{s}$ & This work \\
\hline
\end{tabular}

Table 2 Comparisons of the critical parameters for PTE photodetectors based on perovskite and graphene materials

\begin{tabular}{|c|c|c|c|c|c|}
\hline Description & Wavelength & Responsivity (mA W ${ }^{-1}$ ) & Detectivity (Jones) & Response time & Ref. \\
\hline RGO films & 375 nm-118 $\mu \mathrm{m}$ UV-THz & $87.3-2.8 \mathrm{mV} \mathrm{W}^{-1}$ (0 V bias $)$ & $4.23 \times 10^{6}$ & $34 \mathrm{~ms}$ & 12 \\
\hline EuBiSe $_{3}$ crystal & $405 \mathrm{~nm}-118 \mu \mathrm{m}$ UV-THz & $1.25-0.69 \mathrm{~V} \mathrm{~W}^{-1}$ (0 V bias) & $2.91 \times 10^{8}$ & $207 \mathrm{~ms}$ & 13 \\
\hline 3D MG & $118 \mu \mathrm{m} \mathrm{THz}$ & $5.1 \mathrm{mV} \mathrm{W}^{-1}$ (0 V bias) & $2.5 \times 10^{5}$ & $23 \mathrm{~ms}$ & 41 \\
\hline $\mathrm{MAPbI}_{3} / \mathrm{PEDOT}: \mathrm{PSS}$ & $1.064-118 \mu \mathrm{m}$ NIR-THz & $1.6 \times 10^{-3}(0 \mathrm{~V}$ bias $)$ & $1.2 \times 10^{7}$ & $28 \mu \mathrm{s}$ & This work \\
\hline
\end{tabular}

of the $\mathrm{MAPbI}_{3} / \mathrm{PEDOT}$ :PSS device is extended to the $\mathrm{THz}$ range, which is advantageous for THz detection.

\section{Conclusions}

In summary, we proposed a fast response self-powered NIR and THz PTE PD that was assembled using a structure of ITO/ PEDOT:PSS/MAPbI ${ }_{3} / \mathrm{Au}$. By constructing the PEDOT:PSS/MAPbI ${ }_{3}$ junction, the enhanced Seebeck coefficient and high electrical conductivity were investigated. Subsequently, the photoresponse and response speed of the device were optimized under $1064 \mathrm{~nm}$ and 2.52 THz irradiation. Furthermore, the PTE PD shows a high response speed of $28 \mu \mathrm{s}$ with a $-3 \mathrm{~dB}$ bandwidth of $5 \mathrm{kHz}$. These results indicate that the PEDOT:PSS/MAPbI ${ }_{3}$ composition is a good candidate material for fabricating high speed, self-powered and room temperature operation NIR and $\mathrm{THz}$ optoelectronic devices.

\section{Experimental details}

\section{Synthesis of $\mathrm{MAPbI}_{3}$}

The $\mathrm{MAPbI}_{3}$ thin film was grown by using an antisolvent method. The $\mathrm{MAPbI}_{3}$ perovskite precursor solution was prepared by dissolving a mixture of $\mathrm{PbI}_{2}(99.99 \%)$ and MAI (99\%) by using a molar ratio of $1: 1$. The mixture dissolved in a mixed solvent DMF $(99.5 \%)$ and DMSO (99.5\%) with a volume ratio of $7: 3$. The precursor solution was spin-coated on the substrate with a rotate speed of $6000 \mathrm{rpm}$. After a delay time of $40 \mathrm{~s}, 150 \mu \mathrm{L}$ of the antisolvent CB was dripped onto the films. Then, the film was annealed in a glove box at $100{ }^{\circ} \mathrm{C}$ for $40 \mathrm{~s}$.

\section{Fabrication process of the Au/MAPbI ${ }_{3} /$ PEDOT:PSS/ITO PD}

The $\mathrm{Au} / \mathrm{MAPbI}_{3} / \mathrm{PEDOT}$ :PSS/ITO PDs were fabricated as follows. A quartz glass sheet coated with an indium tin oxide (ITO) film served as the substrate. The ITO/glass substrate with dimensions of $14 \times 14 \times 2 \mathrm{~mm}$ was handled within a UV-ozone system for $15 \mathrm{~min}$. Then, a PEDOT:PSS aqueous solution (bought from Xi'an Polymer Light Technology Corp) was deposited onto the substrate via spin coating at a rotation speed of $5000 \mathrm{rpm}, 50 \mathrm{~s}$. Subsequently, the PEDOT:PSS film was heated at $150{ }^{\circ} \mathrm{C}$ for $30 \mathrm{~min}$ and was then transferred into a glove box. The $\mathrm{MAPbI}_{3}$ perovskite film was then spin-coated onto the ITO/PEDOT:PSS film substrate. After the coating process was complete, the samples were placed in a vacuum chamber with a vacuum of $10^{-4} \mathrm{~Pa}$. The electrodes were then prepared by the thermal evaporation method on the $\mathrm{MAPbI}_{3}$ perovskite film from $\mathrm{Cr} / \mathrm{Au}(10 / 200 \mathrm{~nm})$ layers using a shadow mask.

\section{Characterization and testing}

The device's cross and surface morphologies were examined by using scanning electron microscopy (SEM) and Atomic Force Microscopy (AFM). The structure of the device was obtained by X-Ray Powder Diffraction (XRD). The photoluminescence (PL) spectrum was recorded using $374 \mathrm{~nm}$ laser. The $I-V$ characteristics and the photocurrents of the device were tested by using a Keithley 2400 source meter with Labview software. The light sources were $1064 \mathrm{~nm}$ semiconductor lasers and a $118 \mu \mathrm{m}$ terahertz source (FIRL 100). The light power was measured by using a power meter with an RS232 port (Ophir Vega). The broadband absorption spectra of the device were recorded using a Zolix Omni- $\lambda 3007$ spectrophotometer with $\mathrm{Si}$ and InGaSn photodetectors. The absorption performance of $\mathrm{MAPbI}_{3}$ in the $\mathrm{THz}$ range was tested using a terahertz time-domain spectroscopy system. The temperature images of the device were obtained by using an Infrared thermal imager (FLIR T630sc). The Seebeck coefficient was measured by using a self-built test system. A heating band was used to produce a temperature difference of the channel. The voltage difference $(\Delta V)$ between the two electrodes was measured by using a Keithley 2400 source meter and the temperature distribution was obtained by using the Infrared thermal imager. The Seebeck coefficient was calculated as $S=\Delta V / \Delta T$, where $\Delta T=T_{\text {hot }}-T_{\text {cold }}$, meaning the temperature difference between the hot side and cold side.

\section{Conflicts of interest}

There are no conflicts to declare. 


\section{Acknowledgements}

This work was supported by the National Natural Science Foundation of China (No. 61675147, 61735010 and 91838301), the National Key Research and Development Program of China (No. 2017YFA0700202), Basic Research Program of Shenzhen (JCYJ20170412154447469), and Beiyang yong junior faculties of Tianjin university (No. 2019XRG-0056).

\section{References}

1 Y. Cao, J. Zhu, J. Xu, J. He, J. L. Sun, Y. Wang and Z. Zhao, Small, 2014, 10, 2345-2351.

2 Y. Liu, J. Yin, P. Wang, Q. Hu, Y. Wang, Y. Xie, Z. Zhao, Z. Dong, J.-L. Zhu, W. Chu, N. Yang, J. Wei, W. Ma and J.-L. Sun, ACS Appl. Mater. Interfaces, 2018, 10, 36304-36311.

3 Y. Z. Yifan Li, Y. Yu, Z. Chen, Q. Li, T. Li, J. Li, H. Zhao, Q. Sheng, F. Yan, Z. Ge, Y. Ren, Y. Chen and J. Yao, Photonics Res., 2020, 8, 368-374.

4 H. C. Zhiyu Huang, Y. Huang, Z. Ge, Y. Zhou, Y. Yang, P. Xiao, J. Liang, T. Zhang, Q. Shi, G. Li and Y. Chen, Adv. Funct. Mater., 2017, 28, 8.

5 C. Liu, L. Wang, X. Chen, J. Zhou, W. Hu, X. Wang, J. Li, Z. Huang, W. Zhou, W. Tang, G. Xu, S.-W. Wang and W. Lu, Carbon, 2018, 130, 233-240.

6 M. S. Vitiello, D. Coquillat, L. Viti, D. Ercolani, F. Teppe, A. Pitanti, F. Beltram, L. Sorba, W. Knap and A. Tredicucci, Nano Lett., 2012, 12, 96-101.

7 Y. Chen, X. Wang, G. Wu, Z. Wang, H. Fang, T. Lin, S. Sun, H. Shen, W. Hu, J. Wang, J. Sun, X. Meng and J. Chu, Small, 2018, 14, 1703293.

8 Q. Han, T. Gao, R. Zhang, Y. Chen, J. Chen, G. Liu, Y. Zhang, Z. Liu, X. Wu and D. Yu, Sci. Rep., 2013, 3, 3533.

9 C. C. E. Peytavit and J.-F. Lampin, J. Appl. Phys., 2011, 109, 016101.

10 X. Xu, N. M. Gabor, J. S. Alden, A. M. Van Der Zande and P. L. Mceuen, Nano Lett., 2010, 10, 562-566.

11 F. H. Koppens, T. Mueller, P. Avouris, A. C. Ferrari, M. S. Vitiello and M. Polini, Nat. Nanotechnol., 2014, 9, 780-793.

12 Y. N. Jianguo Wen, P. Wang, M. Chen, W. Wu, Y. Cao, J.-L. Sun, M. Zhao, D. Zhuang and Y. Wang, Carbon, 2019, 153, 274-284.

13 Y. N. Yingxin Wang, M. Chen, J. Wen, W. Wu, Y. Jin, D. Wu and Z. Zhao, ACS Photonics, 2019, 6, 895-903.

14 M. Buscema, M. Barkelid, V. Zwiller, H. S. Van Der Zant, G. A. Steele and A. Castellanos-Gomez, Nano Lett., 2013, 13, 358-363.

15 S. Limpert, A. Burke, I. J. A. Chen, N. S. Lehmann, S. Fahlvik, S. Bremner, G. Conibeer, C. Thelander, M. E. Pistol and H. Linke, Nano Lett., 2017, 17, 4055-4060.

16 A. V. Emelianov, D. Kireev, A. Offenhäusser, N. Otero, P. M. Romero and I. I. Bobrinetskiy, ACS Photonics, 2018, 5, 3107-3115.
17 X. Cai, A. B. Sushkov, R. J. Suess, M. M. Jadidi, G. S. Jenkins, L. O. Nyakiti, R. L. Myers-Ward, S. Li, J. Yan, D. K. Gaskill, T. E. Murphy, H. D. Drew and M. S. Fuhrer, Nat. Nanotechnol., 2014, 9, 814-819.

18 Y. F. Li, Y. T. Zhang, Z. Chen, Q. Li, T. Li, M. Li, H. Zhao, Q. Sheng, W. Shi and J. Q. Yao, Photon. Res., 2020, 8, 1301-1308.

19 N. Alwadai, M. A. Haque, S. Mitra, T. Flemban, Y. Pak, T. Wu and I. Roqan, ACS Appl. Mater. Interfaces, 2017, 9, 37832-37838.

20 F. Huang, Y. Peng and G. Liu, J. Phys. Chem. C, 2019, 123, 11073-11080.

21 S. Tong, J. Sun, C. Wang, Y. Huang, C. Zhang, J. Shen, H. Xie, D. Niu, S. Xiao, Y. Yuan, J. He, J. Yang and Y. Gao, Adv. Electron. Mater., 2017, 3, 1700058.

22 M. Kim, G.-H. Kim, T. K. Lee, I. W. Choi, H. W. Choi, Y. Jo, Y. J. Yoon, J. W. Kim, J. Lee, D. Huh, H. Lee, S. K. Kwak, J. Y. Kim and D. S. Kim, Joule, 2019, 3, 2179-2192.

23 S. S. Shin, S. J. Lee and S. I. Seok, Adv. Funct. Mater., 2019, 29, 1900455.

24 Y. Li, Y. Zhang, T. Li, M. Li, Z. Chen, Q. Li, H. Zhao, Q. Sheng, W. Shi and J. Yao, Nano Lett., 2020, DOI: 10.1021/acs.nanolett. 0c00082.

25 A. Pisoni, J. Jacimovic, O. S. Barisic, M. Spina, R. Gaal, L. Forro and E. Horvath, J. Phys. Chem. Lett., 2014, 5, 2488-2492.

26 Y. Xiong, L. Xu, P. Wu, L. Sun, G. Xie and B. Hu, Adv. Funct. Mater., 2019, 29, 1900615.

27 J. T. W. Y. Mingyu Zhang, Carbon, 2020, 156, 339-345.

28 K. C. Choongho Yu, L. Yin and J. C. Grunlan, ACS Nano, 2011, 5, 7885-7892.

29 N. Massonnet, A. Carella, O. Jaudouin, P. Rannou, G. Laval, C. Celle and J.-P. Simonato, J. Mater. Chem. C, 2014, 2, 1278-1283.

30 T.-C. Tsai, H.-C. Chang, C.-H. Chen and W.-T. Whang, Org. Electron., 2011, 12, 2159-2164.

31 C. Xieand and F. Yan, ACS Appl. Mater. Interfaces, 2017, 9, 1569-1576.

32 C. Xieand and F. Yan, J. Mater. Chem. C, 2018, 6, 1338-1342.

33 Y. S. Lai, C. Y. Tsai, C. K. Chang, C. Y. Huang, V. K. Hsiao and Y. O. Su, Adv. Mater., 2016, 28, 2644-2648.

34 L. Jin, Y. Zhang, Y. Yu, Z. Chen, Y. Li, M. Cao, Y. Che and J. Yao, Adv. Opt. Mater., 2018, 6, 1800639.

35 F.-X. Liang, J.-Z. Wang, Z.-X. Zhang, Y.-Y. Wang, Y. Gao and L.-B. Luo, Adv. Opt. Mater., 2017, 5, 1700654.

36 H. Zhao, Y. Zhang, T. Li, Q. Li, Y. Yu, Z. Chen, Y. Li and J. Yao, Nanotechnology, 2020, 31, 035202.

37 Q. Zheng, J. Huang, C. Han and Y. Chen, IEEE Electron Device Lett., 2017, 38, 79-82.

38 F. Yi, Q. Liao, Y. Huang, Y. Gu and Y. Zhang, Semicond. Sci. Technol., 2013, 28, 105023.

39 C. Xie, P. You, Z. Liu, L. Li and F. Yan, Light: Sci. Appl., 2017, 6, e17023.

40 S. Dhar, T. Majumder, P. Chakraborty and S. P. Mondal, Org. Electron., 2018, 53, 101-110.

41 Y. W. Meng Chen, J. Wen, H. Chen, W. Ma, F. Fan, Y. Huang and Z. Zhao, ACS Appl. Mater. Interfaces, 2019, 11, 6411-6420. 